\title{
Editorial
}

\section{Robotic Prosthetic Limbs}

\author{
Ruwan Gopura $\mathbb{D}^{1},{ }^{1}$ Kazuo Kiguchi, ${ }^{2}$ George Mann $(\mathbb{D}),{ }^{3}$ and Diego Torricelli ${ }^{4}{ }^{4}$ \\ ${ }^{1}$ Department of Mechanical Engineering, University of Moratuwa, Moratuwa, Sri Lanka \\ ${ }^{2}$ Department of Mechanical Engineering, Kyushu University, Fukuoka, Japan \\ ${ }^{3}$ Faculty of Applied Science, Memorial University of Newfoundland, St. John's, NL, Canada \\ ${ }^{4}$ Cajal Institute, Spanish National Research Council (CSIC), Madrid,, Spain \\ Correspondence should be addressed to Ruwan Gopura; gopura@mech.mrt.ac.lk
}

Received 2 January 2018; Accepted 3 January 2018; Published 1 April 2018

Copyright (c) 2018 Ruwan Gopura et al. This is an open access article distributed under the Creative Commons Attribution License, which permits unrestricted use, distribution, and reproduction in any medium, provided the original work is properly cited.

In the present society, more than one million annual limb amputations are carried out globally due to accidents, war casualties, cardiovascular disease, tumors, or congenital anomalies. Robotic prosthetic limb is a well-established research area that integrates advanced mechatronics, intelligent sensing, and control for achieving higher order lost sensorimotor functions while maintaining the physical appearance of amputated limb. Robotic prosthetic limbs are expected to replace the missing limbs of an amputee restoring the lost functions and providing aesthetic appearance. The main aspects are enhanced social interaction, comfortable amputee's life, and productive amputee to the society. With the advancement of sensor technology, in the last few decades significant contributions have been made in this area. Much of the work is still in the research stage and more research and development work is anticipated in the coming years where the ultimate goal is to produce a device which can generate human-like motions.

This special issue is dedicated to establishing a multidisciplinary forum of discussion on the recent advances in robotic prosthetic limbs. Five articles have been selected to be published in this special issue. Two of them are on upperlimb prosthetic limbs and the other three are on lower-limb prostheses.

One of the articles is on upper-limb robotic prostheses. It proposes a multi-DoF hybrid powered transradial robotic prosthesis. Most hybrid prostheses use individual and decoupled joint control and use either electric or bodypower actuation. Authors of this article have contributed to develop a robotic prosthesis named HyPro. It uses hybrid powering concept on restoration of grasp functions similar to a biological hand. HyPro is an underactuated robot with 15 degrees of freedom and can achieve five grasping patterns: power grasp, tip grasp, lateral grasp, hook grasp, and index point. The underactuated mechanism can achieve required hand preshaping for a given grasping pattern using electricpower in the pregrasp stage and body power is used in grasp stage to execute the final grasping action.

Another article is on transradial prosthetic limbs and it proposes a networked multimodel control method for a prosthesis having $2 \mathrm{DOF}$ wrist and $1 \mathrm{DOF}$ hand. The objective is to enhance the wrist controllability while processing information derived from the joint movement. Authors have applied a manipulative skill model of prehension and it is constrained by forearm properties, grasping object properties, and task. Experimental results confirm the fusion control is sufficient to control the wrist joint with respect to the work plane posture.

It is important to have adaptive capabilities in foot prosthesis to achieve the essential movements of lower-limb amputees as the natural foot enables to do. The authors one article have carried out a systematic review in order to understand the design concepts of adaptive foot prostheses using PRISMA method. Additionally, they have investigated requirements and design challenges of adaptive foot prostheses. Furthermore, adaptive foot prostheses have been classified and compared. Authors have foretold that future adaptive prostheses will consist of energy regenerative methods and will be more convenient for users.

Another article is on a transtibial robotic prosthetic limb. It presents a powered biarticular transtibial prosthesis, which is a combination of a commercial powered ankle-foot 
prosthesis and a motorized robotic knee orthosis. The orthosis is controlled to emulate the human gastrocnemius based on neuromuscular models of matched nonamputees. Authors have evaluated the biarticular condition against the monoarticular condition under the condition that orthosis is behaving as a free-joint. The experimental results obtained from six participants with transtibial amputation do not completely support authors' hypothesis that metabolism decreases for all participants. However, some participants demonstrate large metabolic reductions with the biarticular condition. From the preliminary results it can be suggested that a powered artificial gastrocnemius may be capable of providing large metabolic reductions as compared to a monoarticular prosthesis.

Authors of one of the articles have developed and evaluated a prosthesis that actuates both knee and ankle joints. The prosthesis employs a quasi-passive clutched-spring knee orthosis, approximating the largely isometric behavior of the biological gastrocnemius. Two participants with unilateral transtibial amputation walk with the prosthesis on an instrumented treadmill while motion, force, electromyography, and metabolic data are collected and analyzed. The biarticular system is shown to reduce both affected-side knee and hip moment impulse and positive mechanical work in both participants during the late-stance knee flexion phase of walking, compared to the monoarticular condition. Based on the preliminary results authors have suggested that biarticular functionality may provide benefits beyond even those of the most advanced monoarticular prostheses.

Authors of all articles highlighted both the promise and the challenges faced by the field of robotic prosthetic limbs. Their articles further identified the critical need for additional prospective, in design and control of robotic prosthetic limbs. In summary, this special issue provides a snapshot on the current status of transradial and transtibial robotic prosthetic limbs.

Ruwan Gopura

Kazuo Kiguchi

George Mann

Diego Torricelli 


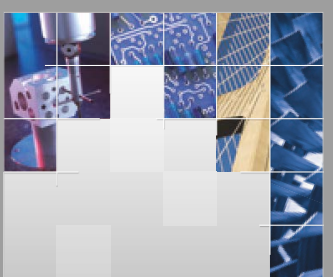

\section{Enfincering}
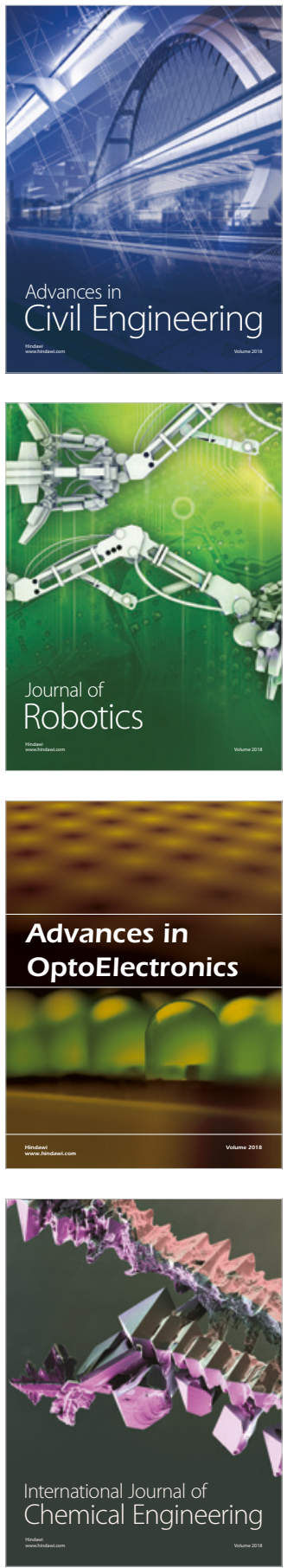

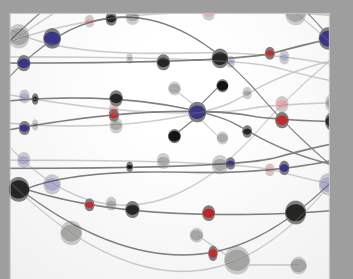

\section{Rotating \\ Machinery}

The Scientific World Journal

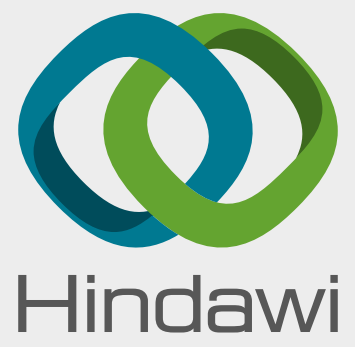

Submit your manuscripts at

www.hindawi.com
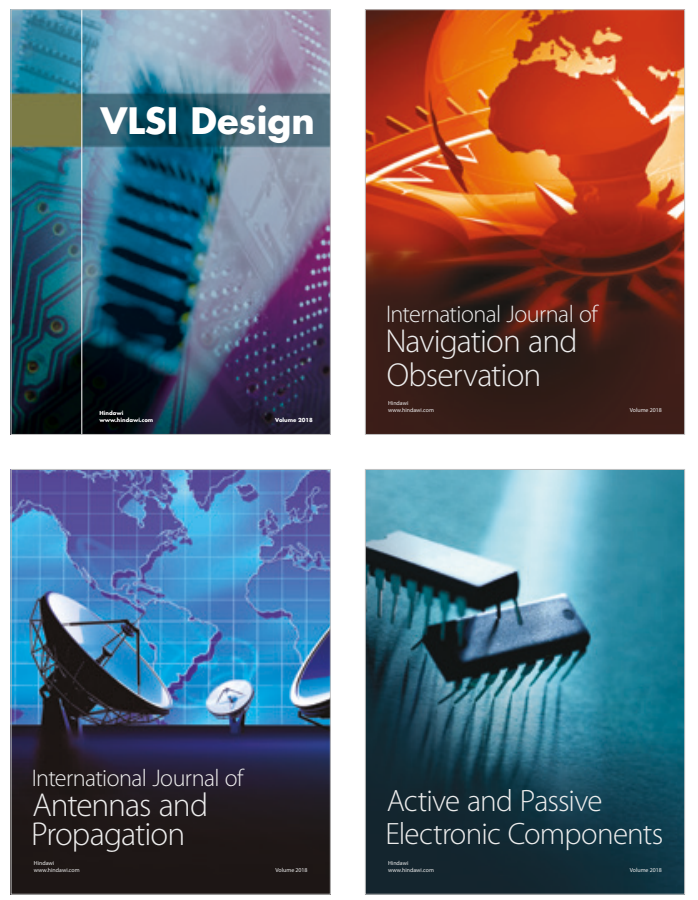
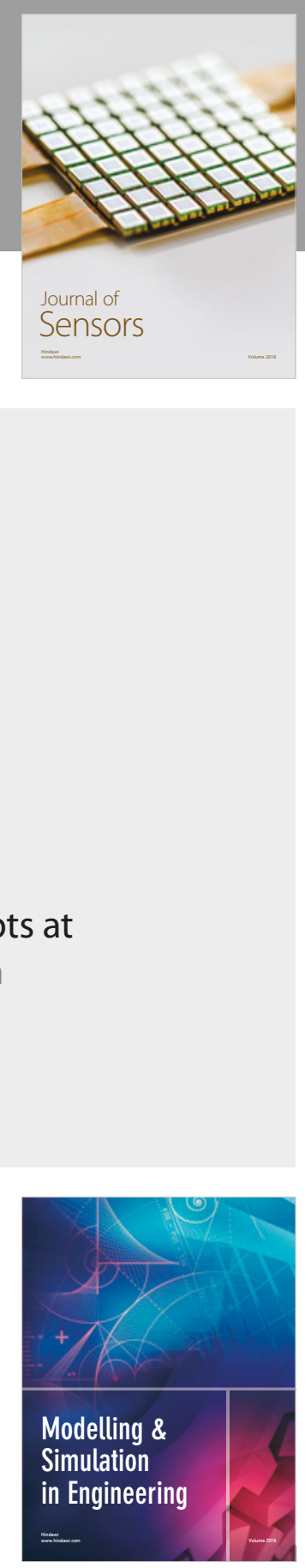

\section{Advances \\ Multimedia}
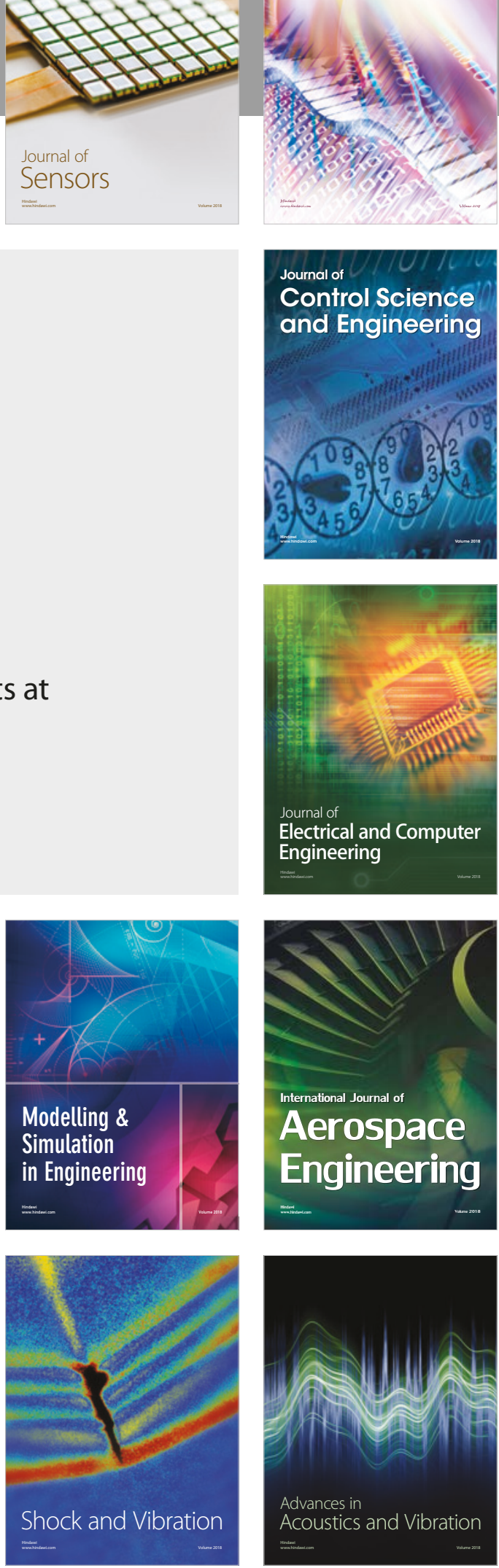